\title{
An Assessment of Echocardiography as a Diagnostic Tool for Dilated Cardiomyopathy in Turkey (Meleagris gallopavo)
}

\author{
${ }^{1}$ Kwaku Gyenai, ${ }^{1}$ Davida Kamara, ${ }^{1}$ Tuoyu Geng, \\ ${ }^{2}$ Robert Lee-Pyle, ${ }^{2}$ Frank Pierson, ${ }^{2}$ Calvert Larsen and ${ }^{1}$ Edward Smith \\ ${ }^{1}$ Department of Animal and Poultry Sciences, \\ ${ }^{2}$ Department of Biomedical Sciences and Pathiobiology, \\ Virginia Polytechnic Institute and State University, Blacksburg, VA 24061, USA
}

Received 2012-07-11, Revised 2012-07-19; Accepted 2012-08-15

\begin{abstract}
Our understanding of the etiology of Dilated Cardiomyopathy (DCM), which affects about $5 \%$ of turkeys, is limited. This limitation may be due to the lack of an easy-to-use diagnostic tool with well-defined parameters and does not involve necropsy. This lack of a widely tested non-necropsy method makes it difficult for a largescale study of the genetic factors that underlie DCM. Here, we Evaluated Echocardiography (ECHO) for its ease and reliability for identifying DCM-affected turkeys from hatch to four weeks-of-age. The parameters evaluated included Left Ventricular Internal-Diastolic (LVIDd), Internal-Systolic Dimension (LVISd), Interventricular Septum End-Diastolic (IVSEd), Interventricular Septum End-Systolic (IVSEs), Left Ventricular Wall End-Systolic (LVWEs) and Left Ventricular Wall End-Diastolic (LVWEd). To induce DCM, feed containing 700 ppm of Furazolidone (Fz) was fed to turkey poults from one to 28 days-of-age. The LVIDd and LVISd were the most consistent indicators of DCM. Both parameters revealed differences between control and treatment poults of between 25 and $326 \%$ at the 4 ages at which ECHO measurements were taken. The average difference in LVIDd between control and poults fed Fz-containing diets ranged from $25 \%$ in one week-old to $80 \%$ in 4 -week-old poults. At similar ages, average differences between control and poults fed Fz-containing diets in LVISd were 74 and 326\% respectively. Necropsy of poults that survived to the end of the 4-week Fz-treatment confirmed these ECHO measurements in treatment and normal poults. Our data suggest that using LVIDd and LVISd as parameters make ECHO a reliable tool for identifying DCM in turkeys.
\end{abstract}

Keywords: Echocardiography, Dilated Cardiomyopathy, Furazolidone, Turkey

\section{INTRODUCTION}

Continued increase in the growth of the turkey industry is due to the increasing consumer demand for turkey as a non-holiday meat item and the use of genetic and non-genetic approaches to increase growth rate and body weight of commercial turkeys (Frame et al., 1999). The gains in production characteristics appear to have been made at the expense of other important physiological traits leading to increased susceptibility to diseases such as Dilated Cardiomyopathy (DCM) or Round Heart Disease (RHD) (Frame et al., 1999).
In commercial turkeys, RHD is believed to be responsible for almost $10 \%$ of poult mortality from hatch to 4 weeks-of-age (Frame et al., 1999). Despite this economic loss to the turkey industry, the etiology of RHD remains poorly understood (The Merck Veterinary Manual, online version, 2004). Understanding the etiology of RHD in the turkey may also contribute to our understanding of human DCM because of the reported similarities of the turkey and human heart (Genao et al., 1996).

Diagnostic tools that have been used to identify turkeys with DCM have primarily been necropsy and to

Corresponding Author: Kwaku Gyenai, Department of Animal and Poultry Sciences, Virginia Polytechnic Institute and State University, Blacksburg, VA 24061, USA 
a limited extent, echocardiography and electrocardiography (Hunsaker, 1971; Czarnecki, 1980; Czarnecki and Good, 1980; Wideman et al., 1998). However, necropsy and electrocardiography are often of limited practical use in the field (The Merck Veterinary Manual, online version, 2004: Accessed July 2012). Necropsy, for example, cannot be used for investigations that involve multi-generational studies including parentoffspring correlation analysis. On the other hand, electrocardiogram is expensive and can only be efficiently used for older birds often past the age that they are most susceptible to DCM. Though Echocardiography (ECHO) has previously been used as a diagnostic tool in birds at or around 4 weeks-of-age, it remains unclear which ECHO parameters are most efficient for the identification of DCM-affected birds from 0 to 4 weeks-of-age (Wu et al., 2004). To be useful, ECHO requires the establishment of baseline parameters that can be referenced in the diagnosis of the incidence and severity of DCM. In the present study, we evaluated the use of ECHO for the diagnosis of DCM in poults from hatch to 4 weeks-of-age, using several ECHO-based measurements including the Left Ventricular Internal-Diastolic (LVIDd), Left Ventricular Internal-Systolic (LVISd), Interventricular Septum EndDiastolic (IVSEd), Interventricular Septum End-Systolic (IVSEs), Left Ventricular Wall End-Systolic (LVWEs) and Left Ventricular Wall End-Diastolic (LVWEd).

\section{MATERIALS AND METHODS}

\subsection{Animals}

Fifty day-old poults obtained from a commercial hatchery were used. The poults were randomly divided into a control and a treatment group of 25 each and raised according to standard protocols (Nesheim et al., 1986). The treatment group was fed a standard turkey poult diet containing 700 parts per million furazolidone (Gwathmey et al., 1999; Liao et al., 1996; 1997). Both groups of poults were fed ad libitum throughout the four-week study. Body weight was recorded weekly for all poults.

\subsection{Diagnosis of DCM}

A portable Aloka ECHO machine with a $7.5 \mathrm{MHz}$ transducer was used to obtain weekly readings in the M-Mode of heart measurements on unsedated, resting animals. The poults were restrained laying on the right side and a transducer with coupling gel was placed inbetween the intercostal cartilage. The rational for using the M-Mode is that it generates a one-dimensional view of small portions of the heart which allow for the detection of axial motion of structures parallel to the beam (Nyland and Mattoon, 1995). The dimensions included in the measurements were the Left Ventricular
End-Diastolic (LVIDd), Left Ventricular End-Systolic (LVISd), Interventricular Septum End-Diastolic (IVSEd), Interventricular Septum End-Systolic (IVSEs), Left Ventricular Wall End-Systolic (LVWEs) and Left Ventricular Wall End-Diastolic (LVWEd).

\subsection{Shortening Fraction}

The in vivo contractile performance of the heart of each poult was estimated according to $\mathrm{Wu}$ et al. (2004) using the equation:

\section{$[($ LVIDd-LVISd)/LVIDd) $] \times 100$}

The rationale is that the shortening fraction estimates the left ventricular performance based on the ratio of the change in the diameter of the left ventricle in diastole and systole.

\subsection{Necropsy}

To validate the ECHO diagnosis, poults that survived to 4 weeks-of-age were weighed and euthanized by cervical dislocation after which the hearts were dissected; atria and large vessels removed and the remaining left and right ventricles were trimmed off and weighed separately (Hunsaker et al., 1971). Histopathology was conducted after fixation in $10 \%$ buffered formalin for twenty-four hours. Tissue sections were stained with hematoxylin and eosin and viewed using standard light microscopy.

\subsection{Statistical Analysis}

Statistical analysis was conducted in SAS using General Linear Model (GLM) procedure to evaluate differences between control and Fz-treatment means. The Student t-test was used to evaluate pair wise differences with significance set at $\mathrm{p}<0.05$ (SAS, 2004).

\section{RESULTS}

Echocardiographic (ECHO) measurements of ventricular dimensions and body weight of treatment and control pouls are presented in Table 1. Poults on normal diet were heavier than those on Furazolidone $(\mathrm{Fz})$ containing diets, though the differences were significant $(p<0.05)$ only at 21 and 28 days-of-age. The slower increase in body weight of poults fed the Fz-containing diet appeared to be an early sign of abnormality. Like body weight, some ECHO parameters like LVIDd and LVISd showed differences between poults on normal and Fz-containing diets as early as 7 days-of-age (Table 1). As shown in Table 1, LVIDd of Fz-diet fed poults increased 25, 32, 47 and $80 \%$ higher in week 1, 2, 3 and 4 , respectively, compared to that of control poults. 
Table 1. Average weekly weight and echocardiography measurements in control (CTL) and poults fed Fz-containing diets (TRT)

\begin{tabular}{|c|c|c|c|c|c|c|c|c|c|c|c|c|c|c|}
\hline \multirow[b]{2}{*}{ Week } & \multicolumn{2}{|c|}{ Body weight(g) } & \multicolumn{2}{|c|}{$\operatorname{LVIDd}(\mathrm{cm})$} & \multicolumn{2}{|c|}{ LVISd $(\mathrm{cm})$} & \multicolumn{2}{|c|}{$\operatorname{IVSEd}(\mathrm{cm})$} & \multicolumn{2}{|c|}{ IVSEs $(\mathrm{cm})$} & \multicolumn{2}{|c|}{ LVWEs $(\mathrm{cm})$} & \multicolumn{2}{|c|}{ LVWEd(cm) } \\
\hline & CTL & TRT & CTL & TRT & CTL & TRT & CTL & TRT & CTL & TRT & CTL & TRT & CTL & TRT \\
\hline$\overline{1}$ & $137.16^{\mathrm{a}}$ & $133.04^{\mathrm{a}}$ & $0.40^{\mathrm{a}}$ & $0.50^{\mathrm{b}^{*}}$ & $0.15^{\mathrm{a}}$ & $0.26^{b^{*}}$ & $0.19^{\mathrm{a}}$ & $0.17^{\mathrm{a}}$ & $0.29^{\mathrm{a}}$ & $0.26^{\mathrm{a}}$ & $0.27^{\mathrm{a}}$ & $0.25^{\mathrm{a}}$ & $0.16^{\mathrm{a}}$ & $0.14^{\mathrm{a}}$ \\
\hline 2 & $303.61^{\mathrm{a}}$ & $281.03^{\mathrm{a}}$ & $0.46^{\mathrm{a}}$ & $0.61^{b^{*}}$ & $0.17^{\mathrm{a}}$ & $0.33^{\mathrm{b}^{*}}$ & $0.25^{\mathrm{a}}$ & $0.23^{\mathrm{a}}$ & $0.35^{\mathrm{a}}$ & $0.33^{\mathrm{a}}$ & $0.34^{\mathrm{a}}$ & $0.33^{\mathrm{a}}$ & $0.21^{\mathrm{a}}$ & $0.19^{\mathrm{a}}$ \\
\hline 3 & $521.40^{\mathrm{a}^{*}}$ & $400.44^{b}$ & $0.57^{\mathrm{a}}$ & $0.84^{b^{*}}$ & $0.21^{\mathrm{a}}$ & $0.62^{\mathrm{b}^{*}}$ & $0.25^{\mathrm{a}}$ & $0.33^{\mathrm{b}^{*}}$ & $0.39^{\mathrm{a}}$ & $0.30^{\mathrm{b}}$ & $0.38^{\mathrm{a}}$ & $0.33^{\mathrm{a}}$ & $0.20^{\mathrm{a}}$ & $0.19^{\mathrm{a}}$ \\
\hline 4 & $880.17^{\mathrm{a}^{*}}$ & $564.06^{\mathrm{b}}$ & $0.70^{\mathrm{a}}$ & $1.26^{*}$ & $0.27^{\mathrm{a}}$ & $1.15^{\mathrm{b}^{*}}$ & $0.34^{\mathrm{b}^{*}}$ & $0.17^{\mathrm{a}}$ & $0.48^{\mathrm{b}^{*}}$ & $0.22^{\mathrm{a}}$ & $0.44^{\mathrm{b}^{*}}$ & $0.21^{\mathrm{a}}$ & $0.28^{\mathrm{b}^{*}}$ & $0.16^{\mathrm{a}}$ \\
\hline
\end{tabular}

where LVIDd, LVISd, IVSEd, IVSEs, LVWEs, LVWEd are left ventricular end-diastolic dimension, left ventricular end-systolic dimension, interventricular septum end-diastolic, interventricular septum end-systolic, left ventricular wall end-systolic, left ventricular wall end-diastolic respectively, as determined from ultrasound measurements by echocardiography. ${ }^{\mathrm{a}, \mathrm{b}}$ : Measurements in the same row with different alphabetic superscript are statistically significantly $* \mathrm{p}<0.05$

Table 2. Ventricular and heart weight of control and poults fed Fz-containing (TRT) after necropsy at four weeks-of- age

\begin{tabular}{lllllc}
\hline Week 4 & Body weight $(\mathrm{g})$ & RVW $(\mathrm{g})$ & LVW $(\mathrm{g})$ & WOH $(\mathrm{g})$ & Apex-Thorax $(\mathrm{mm})$ \\
\hline Control (18) & $839.02 \pm 12.00^{\mathrm{a}^{*}}$ & $0.70 \pm 0.13^{\mathrm{a}^{*}}$ & $3.08 \pm 0.48^{\mathrm{a}}$ & $3.87 \pm 0.66^{\mathrm{a}}$ & $21.12 \pm 1.19^{\mathrm{a}}$ \\
Treatment (9) & $545.89 \pm 79.76^{\mathrm{b}}$ & $0.56 \pm 0.18^{\mathrm{b}}$ & $2.67 \pm 0.68^{\mathrm{a}}$ & $3.37 \pm 0.86^{\mathrm{a}}$ & $19.99 \pm 2.22^{\mathrm{a}}$ \\
\hline Wh
\end{tabular}

Where: RVW, LVW and WOH represent the right ventricular weight, left ventricular; weight and whole heart weight, respectively. ${ }^{a, b}$ : Means \pm S.E. with the same superscript are not different. ${ }^{*} p<0.05$

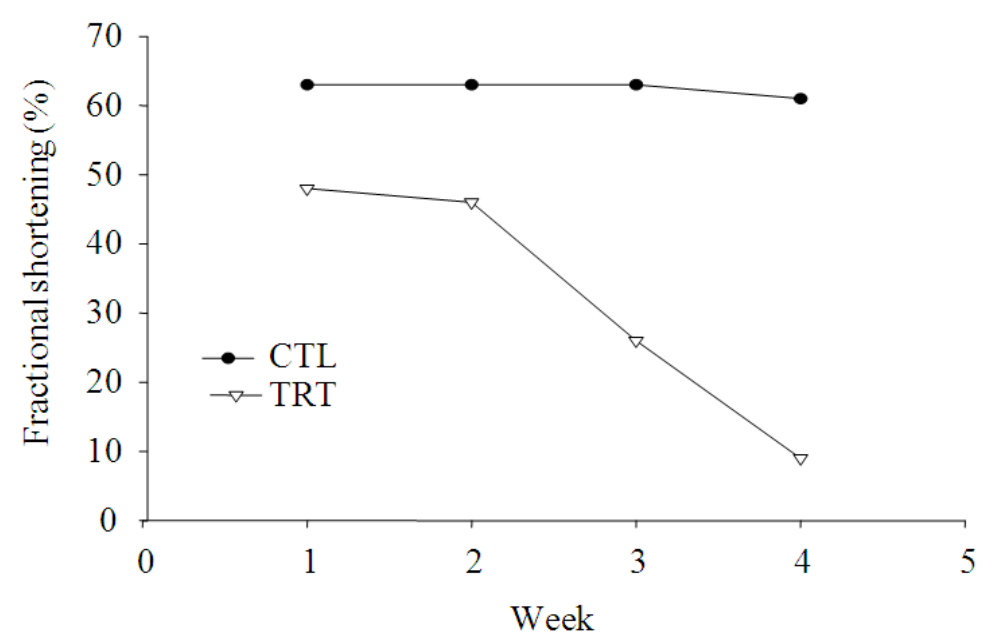

Fig. 1. Fractional shortening (ejection fraction) of the heart from control (CTL) and poults fed Fz-containing diet fed turkey poults (TRT). Significant decrease in TRT poults after 2 weeks-of-age. Means \pm S.E

The LVISd showed a similar though smaller trend. In week 4, the LVISd of Fz-containing diets fed poults was 4-fold larger than those of normal poults (Table 1, Fig. 2 and 3). Similarly, fractional shortening measurment was significantley for poults fed diets containning $\mathrm{Fz}$ when compared to those on normal diets (Fig. 1). Differences were by as much as 20 to $600 \%$ (Fig. 1). This difference in dilation may account for the differences in the weights of the right ventricle, left ventricle, whole heart and the measurement from the apex to the thorax obtained from necropsy (Table 2). The other ECHO parameters evaluated including IVESd, IVSEs, LVWEs and LVWEd showed inconsistent and no significant differences $(p>0.05)$ between poults fed Fz-containing diets and control (Table 1). Echocardiographic IVESD measurements taken at 3 weeks-of-age showed the treatment poults fed Fz-containing diets to have significantly $(p<0.05)$ larger ventricular dimension over the control. While at week 4, poults fed normal diets had significantly larger IVESd, IVSEs, LVWEs and LVWEd (Table 1). In addition to weight and ECHO, further support for the detrimental effects of the toxic diet was the progressive increase in mortality from 7 days-of-age and peaking at 14 days-of-age.

Necropsy measurements validated the ECHO diagnostics of DCM-affected and normal poults. The necropsy showed that the hearts of poults fed Fzcontaining diets had significant dilation of the left ventricle and thinning of the left ventricle free wall and ventricular septum (Table 2). 
Histopathological examination of poults with DCM revealed degeneration of myocytes, B-cells with vacuoles, necrotic cells deep in the left ventricle and extensive inflammation with a significant number of lymphocytes (Fig. 4). In the control group, however, only minimal degeneration of myocytes and necrosis of the left ventricle were apparent (Fig. 5). These observations confirmed the diagnosis made by ECHO in identifying poults as DCM affected or normal. The gross morphological observations were also consistent with characteristics defined by others for DCM-affected poults (Jawad, 1996).



Fig. 2. M-mode echocardiographic image of left ventricle from a poult fed Fz-containing diet at 4 weeks-of-age. The numbers shown on the right hand side used for diagnosis are the left ventricular internal-diastolic and systolic dimension, 2.43 and $2.17 \mathrm{~cm}$, resp



Fig. 3. M-mode echocardiographic image of left ventricle from a poult fed normal diet at 4 weeks-of-age. The numbers shown on the right hand side used for diagnosis are the left ventricular internal-diastolic and systolic dimension, 0.95 and $0.90 \mathrm{~cm}$, resp 




Fig. 4. Organ; Cross section of the heart of a poult fed Fzcontaining diets (diagnosed as dilated cardiomyopathic using echocardiography) showing increased necrosis, epicarditis, vacuolation, as well as significant degeneration of myocytes deep in left ventricle. Hematoxylin and eosin staining. Bar $=10 \mathrm{X} \mu \mathrm{m}$

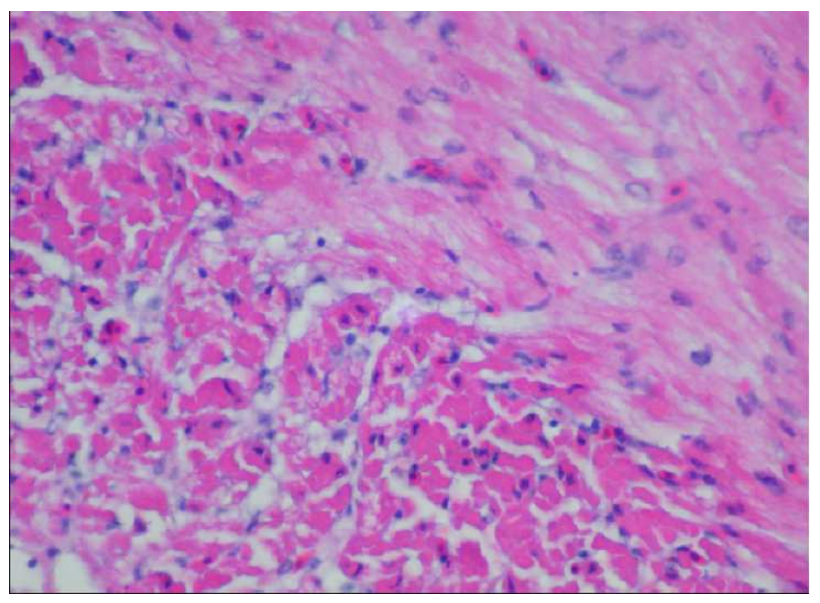

Fig. 5. Organ; Cross section of the heart of control (normal) poult showing small superficial region of vacuolation, some necrosis deep in left ventricle. Hematoxylin and eosin staining. Bar $=10 \mathrm{X} \mu \mathrm{m}$

\section{DISCUSSION}

In this study, we found increased left ventricular measurements LVIDd and LVISd for poults fed Fzcontaining diets compared to control group poults at 4 weeks-of-age. The toxic levels of Fz added to the diets of poults may have resulted to a combination of myocyte injury and necrosis associated with myocardial fibrosis, which led to impaired mechanical function leading to ventricular dilation (Goldstein et al.,
1998). Also addition of Fz may have led to direct toxicity or mechanical insults such as; chronic volume overload in mitral valvular regurgitation with myocyte failure and cytoskeletal uncoupling of the chambers leading to dilation (Goldstein et al., 1998). The differences observed between LVIDd and LVISd for poults fed Fz-containing diets and those in the control group are similar to that of $\mathrm{Wu}$ et al. (2004), which reported increased left ventricular dimension for poults fed Fz-containing diets. More so, a review by Vlahovic and Popovic (1999), reported impaired left ventricular diastolic function to be one of the abnormalities associated with cardiac diseases accompanied by changes in filling patterns in humans, from delayed relaxation, through pseudo-normalization and, finally, to restrictive filling. Left ventricular diastolic dysfunction has also been reported in patients with hypertensive or valvular heart disease as well as in hypertrophic and DCM (Mandinov et al., 2000). Andrade et al. (2005) tested the surgical technique plication of the left ventricular free wall, to reduce left ventricular area and volume and improve left ventricular systolic function, without using a cardiopulmonary bypass in crossbred dogs. They reported plication of the left ventricular free wall to reduce left ventricular area and volume and improve left ventricular systolic function in crossbred dogs with doxorubicin induced cardiomyopathy giving low morbidity and mortality which is similar to the findings in our study.

\section{CONCLUSION}

Here we have shown that ECHO as a diagnostic tool consistently identifies poults with Fz-induced DCM from 2 weeks-of-age, but it can detect the development of DCM in poults as young as 1 week. This can be useful in commercial turkey production and management. Furthermore, the use of ECHO eliminates the impracticality of using necropsy and electrocardiography for the diagnosis of DCM. Though relatively expensive, ECHO will allow diverse investigations including genetic and molecular research to further define the etiology of DCM. ECHO is a relatively easy tool to use and appears to yield measurements of dimensions indicative of DCM that are consistent with those obtained through necropsy.

\section{ACKNOWLEDGEMENT}

We are grateful to the staff of Virginia Tech turkey facility for their help and to Dr. Curtis Novak for his 
help in formulating the ration. Financial support was provided by the Virginia Ag. Council and the NHGRI.

\section{REFERENCES}

Andrade, J.N., A.A. Camacho, P.S. Santos, A.P. Fantinatti and N. Nunes et al., 2005. Plication of the free wall of the left ventricle in dogs with doxorubicin-induced cardiomyopathy. Am. J. Vet. Res., 66: 238-243. PMID: 15757121

Czarnecki, C.M. and A.L. Good. 1980. Electrocardiographic technic for identifying developing cardiomyopathies in young turkey poults. Poult. Sci., 59: 1515-1520. PMID: 7393860

Czarnecki, C.M., 1980. Furazolidone-induced cardiomyopathy-Biomedical model for the study of cardiac hypertrophy and congestive heart failure. Avian Dis., 24: 120-138. DOI: 10.2307/1589772

Frame, D.D., R.E. Buckner and G.L. Anderson, 1999. Causes and control of spontaneous cardiomyopathy or roundheart disease in utah Turkeys. Electrical Publishing.

Genao, A., K. Seth, U. Schmidt, M. Charles and J.A. Gwathmey, 1996. Dilated cardiomyopathy in turkeys: An animal model for the study of human heart failure. Lab. Anim. Sci., 46: 399-403. PMID: 8872990

Goldstein, D.S., J.E. Brush, G. Eisenhofer, R. Stull and M. Esler, 1998. In vivo measurement of neuronal uptake of norepinephrine in the human heart. Circulation, 78: 41-48. PMID: 3383409

Gwathmey, J.K., C.S. Kim, R.J. Hajjar, F. Khan and T.G. DiSalvo et al., 1999. Cellular and molecular remodeling in a heart failure model treated with the beta-blocker carteolol. Am. J. Physiol. Heart Cir. Physiol., 276: H1678-H1690. PMID: 10330254

Hunsaker, W.G., 1971. Round heart disease in four commercial strains of turkeys. Poult. Sci., 50: 1720-1724. PMID: 4258196

Hunsaker, W.G., A. Robertson and S.E. Magwood, 1971. The effect of round heart disease on the electrocardiogram and heart weight of turkey poults. Poult. Sci., 50: 1712-1720. PMID: 4258195

Jawad, I.A., 1996. A Practical Guide to Echocardiography and Cardiac Doppler Ultrasound. 2nd Edn., Lippincott Williams and Wilkins, Boston, ISBN-10: 0316458376, pp: 432.

Liao, R., L. Nascimben, J. Friedrich, J.K. Gwathmey and J.S. Ingwall, 1996. Decreased energy reserve in an animal model of dilated cardiomyopathy Relationship to contractile performance. Am. J. Cir. Res., 78: 893-902. PMID: 8620610
Liao, R., M. Charles and J.K. Gwathmey, 1997. Animal models of cardiovascular disease for pharmacologic drug development and testing: Appropriateness of comparison to the human disease state and pharmacotherapeutics. Am. J. Ther., 4: 149-158. PMID: 10423605

Mandinov, L., F. R. Eberli, C. Seiler and O.M. Hess, 2000. Diastolic heart failure. Cardio. Res., 45: 813825. PMID: 10728407

Nesheim, M. C., R. E. Austic and L. E. Card, 1986. Poultry Production. 12th Edn., Lea and Febiger, Philadelphia.

Nyland, T.G. and J.S. Mattoon, 1995. Veterinary Diagnostic Ultrasound. 1st Edn., WB Saunders, Philadelphia, ISBN-10: 0721627455, pp: 357.

SAS, 2004. Version 8.01, Copyright (C) 2000-2004. SAS Institute Inc. Cary, NC, USA.

Vlahovic, A. and A.D. Popovic, 1999. Evaluation of left ventricular diastolic function using Doppler echocardiography. Med. Pregl., 52: 13-18. PMID: 10352498

Wideman, R.F. Jr., T. Wing, Y.K. Kirby, M.F. Forman and N. Marson et al., 1998. Evaluation of minimally invasive indices for predicting ascites susceptibility in three successive hatches of broilers exposed to cool temperatures. Poult. Sci., 77: 1565-1573. PMID: 9776067

Wu, Y., A.H. Tobias, K. Bell, W. Barry and M. Helmes et al., 2004. Cellular and molecular mechanisms of systolic and diastolic dysfunction in an avian model of dilated cardiomyopathy. J. Mol. Cell. Cardiol., 37: 111-119. PMID: 15242741 